\title{
Non-maximally decimated filter bank-based single-carrier receiver: a pathway to next-generation wideband communication
}

\author{
Xiaofei Chen ${ }^{1 *}$, fred harris ${ }^{1 \dagger}$, Elettra Venosa ${ }^{1+}$ and Bhaskar D Rao ${ }^{2+}$
}

\begin{abstract}
We present the design of a wideband digital modem based on non-maximally decimated filter bank (NMDFB) with perfect reconstruction (PR) property. The PR-NMDFB contains an analysis filter bank (AFB) and a synthesis filter bank (SFB) whose efficient polyphase forms are named as polyphase analysis channelizer (PAC) and polyphase synthesis channelizer (PSC). The waveform being processed is the legacy square root Nyquist-shaped quadrature amplitude modulation (QAM). In contrast to orthogonal frequency division multiplexing (OFDM) systems, the shaped QAM transmission has much superior performance properties in throughput, peak-to-average power ratio (PAPR), and synchronization. We will show the PR-NMDFB is capable of efficiently performing several key tasks of a digital receiver with dramatic workload reduction. This includes digital filtering, carrier recovery, and symbol timing recovery. Moreover, the nature of NMDFB allows the signal processing to operate a significantly reduced sample rate, which is a desired characteristic for replacing current FIR implementation in wideband systems.
\end{abstract}

Keywords: Filter bank; Single-carrier transmission; Perfect reconstruction; Non-maximally decimated filter bank; Synchronization

\section{Introduction}

The wireless technology has experienced significant growth in the past decades; and we have seen generations of wireless communication systems increasing their bandwidth and data rates by more than an order of magnitude per generation. Current systems offer 100-Mbps data rates in $20-\mathrm{MHz}$ bandwidth links. We can expect future generation wireless systems to offer $1 \mathrm{Gbit} / \mathrm{s}$ data rates with $500-\mathrm{MHz}$ bandwidth links. As the demands for high data rate communication grows, the conventional methods, i.e., shaped quadrature amplitude modulation (QAM), become seemingly incapable of dealing with highly dispersive channels in a cost-effective manner. The legacy receivers often require building several time offset overlapped finite impulse response (FIR) filters operating in parallel to perform synchronization, matched filtering, as well as channel equalization. As the

\footnotetext{
* Correspondence: chenxiaofei_sdsu@yahoo.com

${ }^{\dagger}$ Equal contributors

'Department of Electrical and Computer Engineering, San Diego State University, San Diego, CA 92182-1309, USA

Full list of author information is available at the end of the article
}

signal bandwidth grows comparable to the hardware's clock rate, any FIR filtering becomes extremely expensive. Meanwhile, the frequency-selective channel requires very sophisticated equalizers, which further increases the difficulty in fitting the legacy QAM waveform into today's communication systems. To overcome this problem, many broadband techniques [1] such as spread spectrum and multicarrier transmission were introduced. Although the orthogonal frequency division multiplexing (OFDM) technique [2] suffers from problems such as high peak-to-average power ratio (PAPR), sensitivity to carrier frequency offset [3], and time and frequency guard spans, it has become dominant because of its simple equalizer structure for highly frequency-selective channels. An unappreciated advantage of OFDM is its fast Fourier transform (FFT)-based block processing nature [2], which not only takes advantage of FFT's efficiency but also reduces the hardware processing speed, due to the serial/ parallel conversion at the transmitter and receiver.

The modern research towards wideband communication systems can be generally categorized as follows: (1) block-based transmission with cyclic prefix-occupied 
guard interval, which includes the conventional OFDM system and discrete Fourier transform (DFT)-precoded OFDM single-carrier modulation [3-7]; (2) non-blockbased continuous streaming signal format, without cyclic prefix or guard interval, which includes filter bank (FB)based solutions such as filter bank multicarrier offset QAM [8-10] and filter bank single-carrier (FBSC) transmission [11-14]. The FBSC transmission is not well studied compared to FBMC, but it is believed to have significant influence in future high-throughput transmissions such as backhaul modems. In this paper, we shall focus on developing a unified receiver structure for FBSC transmissions. Figure 1 shows a perfect reconstruction non-maximally decimated filter bank (PR-NMDFB)-based FBSC receiver that embeds the carrier frequency offset (CFO) removal, fractional delay filter (FDF) for finding the correct symbol timing, matched filter (MF), and equalization process in between the polyphase analysis channelizer (PAC) and polyphase synthesis channelizer (PSC). These signal processing tasks are traditionally implemented by separate filters in the time domain, and we will show that they can also be implemented in the FB-transformed domain as independent intermediate processing elements (IPEs). Since the signals presented at PAC parallel output ports are highly decimated, the IPE processing demonstrates impressive workload reduction compared to the conventional time domain methods.

Several authors have studied the FBSC from equalization perspectives and have shown the equalization task is closely related to subband adaptive filtering (SAF) [15]. The authors in [12] show the preliminary examples on FBbased channel identification. The authors in [11] demonstrated the design of fractionally spaced equalizer (FSE) via NMDFBs (oversampled FBs). The authors in this paper address the design of FBSC from the synchronization perspective. In particular, we will introduce the recently proposed [16] PR-NMDFB-based digital filtering concept which allows us to carry out much more rigorous performance analysis towards FBSC compared to the past works [11-14]. Note that the PR-NMDFB in practice will become near-perfect reconstruction (NPR) due to practical realization; however, the bounds on reconstruction errors require arithmetic with sufficient bit width to support the stop-band attenuation levels.
We will present the NMDFB-based carrier frequency recovery, symbol timing recovery, and matched filtering (MF) process. These independent tasks were not discussed in the past works on FBSC. The novelty of this paper is as follows: (1) we present the PR-NMDFB filtering-based formulation for FBSC and (2) propose the synchronization techniques for FBSC in the FB-transformed domain. To our best knowledge, these topics were not presented before or studied in depth.

The organization of this paper is as follows: Section 2 introduces the PR-NMDFB and its filtering property, Section 3 presents the carrier frequency recovery technique, Section 4 solves the symbol time recovery problem, Section 5 performs the complexity analysis, Section 6 presents the simulation results, and Section 7 draws the conclusion.

\section{Review of PR-NMDFB and its filtering property}

The basic building blocks of FBSC modems are based on oversampled or non-maximally decimated FBs with PR support, which are in general considered well studied [17-19] and have numerous variations [20-24]. The implementation of FBSC can be viewed using FB to perform digital filtering in the channelizer domain; filtering is achieved by altering the gain and phase over each spectral span presented at analysis filter bank (AFB) outputs. In order to accomplish this FB-based filter task, several restrictions on FB need to be met: (1) it must have PR or near-PR property. (2) The aliasing cancelation should not involve other channels; as a counter example, the cosine-modulated filter bank (CMFB) [24] requires adjacent channels to cancel aliasing thus generally cannot be used in communication systems. (3) Efficient polyphase implementation must exist, preferably with fixed channel size $M$ and adjustable decimation factor $D(D<M)$. One filter bank design that meets these conditions is proposed by Karp, in Figure nine of [20] under the name 'DFT polyphase realization', which serves as an intermediate step to derive the polyphase implementation of modified DFT (MDFT) [20,25].

\subsection{Background on PR-NMDFB}

The theoretical model of a generalized DFT-based PRNMDFB is shown in Figure 2. The AFB contains $M$ band-

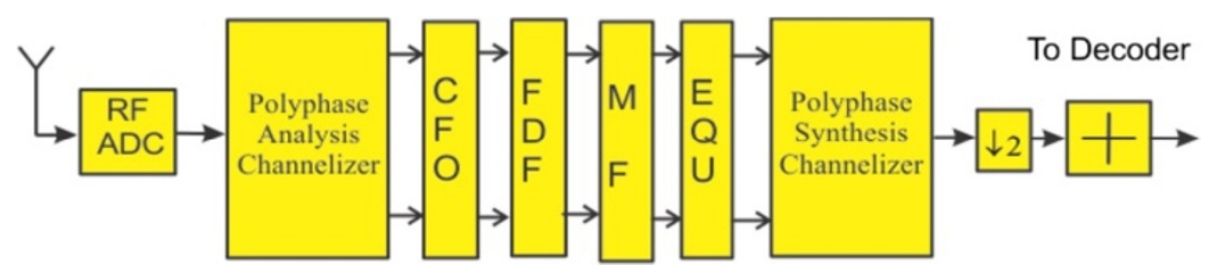

Figure 1 High-level architecture of FBMC receiver. 


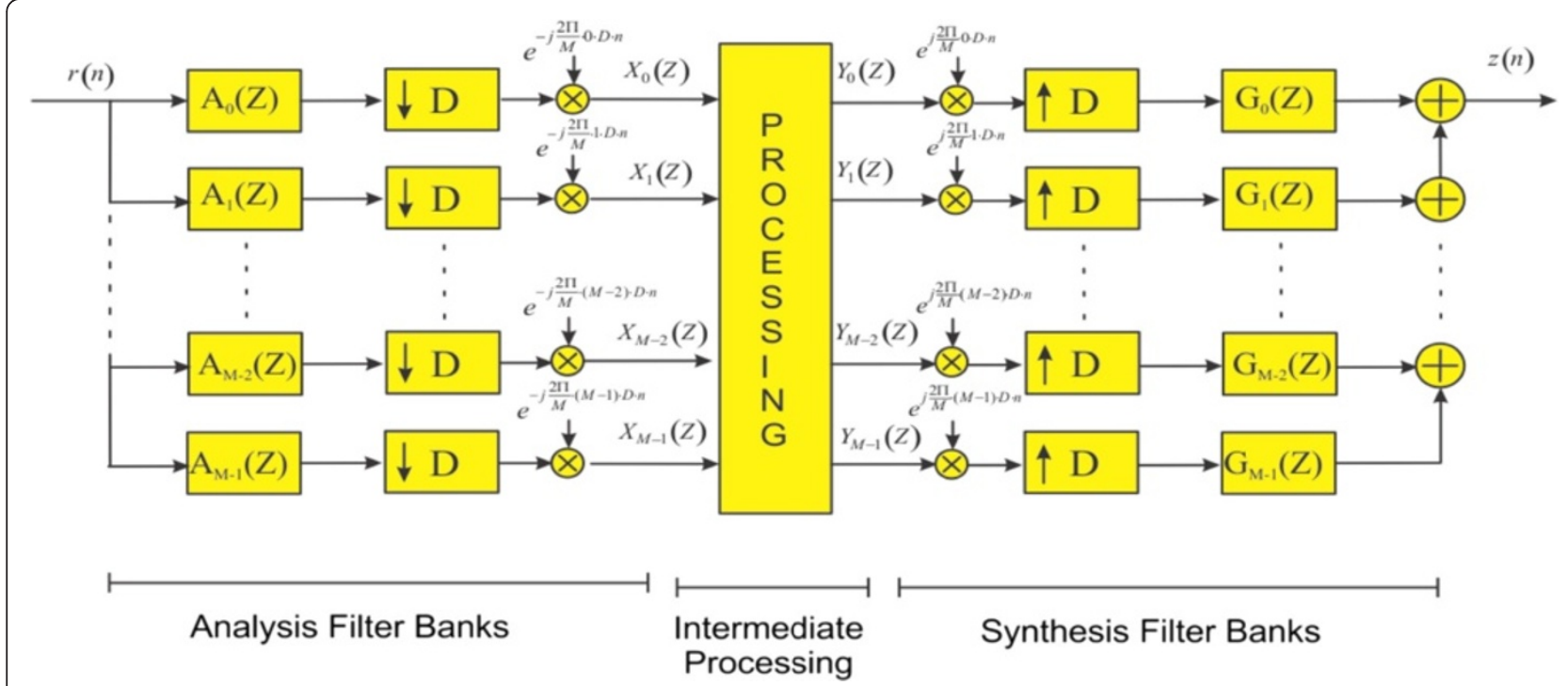

Figure 2 Generalized $M$-path non-maximally decimated filter bank model.

pass filters (BPFs), whose $Z$-transforms are $A_{m}(Z)$, for $m=0,1, \ldots, M-1$. The $M$ BPFs have equal bandwidth and are centered on digital frequencies $\theta_{m}=\frac{2 \pi}{M} m$, for $m=0,1, \ldots, M-1$. Let $a(n)$ be the impulse response of the low-pass prototype filter (LPPF). The impulse response of the $m$ th BPF is $a_{m}(n)=a(n) e^{j \frac{2 \pi}{M} m n}$, whose $Z$-transform is $A_{m}(Z)=A\left(e^{-j \frac{2 \pi}{M} m} Z\right)=A\left(W_{M}^{m} Z\right)$, and $W_{M} \underline{\underline{\text { def }}} e^{-j \frac{2 \pi}{M}}$. A down-sampling by a factor $D(D<M)$, which is an integer that divides $M$, follows each BPF. As a standard practice, we require the AFB filtered and downsampled signal to be centered on zero frequency, and this is done via a set of complex rotators whose values are $e^{-j \frac{2 \pi}{M} m n D}$. The AFB-transformed signal is then processed by the intermediate processing element denoted as IPE at deeply decimated sampling rate. The synthesis filter bank (SFB) performs the exact inverse processes of AFB: heterodyne, up-sampling, and filtering. It can be shown [16] that the $Z$-transform of the output signal $z(n)$ is

$$
\begin{aligned}
Z(Z) & =\frac{1}{D} \boldsymbol{G}_{1 \times M}^{T}(Z) \mathbb{K}_{M \times M} \mathbb{A}_{M \times D}(Z) \boldsymbol{R}_{D \times 1}(Z) \\
& =\frac{1}{D} \boldsymbol{T}_{1 \times D}^{\mathbb{K}}(Z) \boldsymbol{R}_{D \times 1}(Z) \\
& =\frac{1}{D} T_{s}^{\mathbb{K}}(Z) R(Z)+\frac{1}{D} \boldsymbol{T}_{A}^{\mathbb{K}}(Z) \overline{\boldsymbol{R}}(Z)
\end{aligned}
$$

where $G(Z)=\left[G\left(Z W_{M}^{0}\right) \ldots G\left(Z W_{M}^{M-1}\right)\right]^{T}$ are the BPFs for SFB, $\mathbb{K}_{M \times M}=\operatorname{diag}\left\{K_{0} \ldots K_{M-1}\right\}$ is the IPE complex scalar gain applied in between AFB and SFB, column vector $R(Z)=\left[R\left(Z W_{D}^{0}\right) \ldots R\left(Z W_{D}^{D-1}\right)\right]^{T}=\left[R\left(Z W_{D}^{0}\right) \bar{R}(Z)\right]^{T}$ is the modulated versions of the input signal, matrix $\mathbb{A}_{M \times D}$ $(Z)$ is defined as

$$
\begin{aligned}
\mathbb{A}(\boldsymbol{Z}) & =\left[\begin{array}{ccc}
A\left(Z W_{M}^{0} W_{D}^{0}\right) & \ldots & A\left(Z W_{M}^{0} W_{D}^{D-1}\right) \\
\vdots & \ddots & \vdots \\
A\left(Z W_{M}^{M-1} W_{D}^{0}\right) & \ldots & A\left(Z W_{M}^{M-1} W_{D}^{D-1}\right)
\end{array}\right]_{M \times D}, \\
& =\left[\boldsymbol{A}_{M \times 1} \mid \overline{\mathbb{A}}_{M \times(D-1)}\right]_{M \times D}
\end{aligned}
$$

and $\boldsymbol{T}^{\mathbb{K}}{ }_{1 \times D}(\boldsymbol{Z}) \triangleq \boldsymbol{G}_{1 \times M}^{T}(\boldsymbol{Z}) \mathbb{K}_{M \times M} \mathbb{A}_{M \times D}(\boldsymbol{Z})=\left[T_{s}^{\mathbb{K}}(Z) \boldsymbol{T}_{A}^{\mathbb{K}}(Z)\right]$ is the total transfer function (TF) for the $M$-path, decimated by $D$, AFB, and SFB. $T_{s}^{\mathbb{K}}(Z) \triangleq G_{1 \times M}^{T}(Z) \mathbb{K}_{M \times M}$ $\boldsymbol{A}_{M \times 1}(\boldsymbol{Z})$ is the desired signal TF, whereas $\boldsymbol{T}_{A}^{\mathbb{K}}(Z) \triangleq \boldsymbol{G}_{1 \times M}^{T}$ $(\boldsymbol{Z}) \mathbb{K}_{M \times M} \overline{\mathbb{A}}_{M \times(D-1)}(\boldsymbol{Z})$ is the undesired aliasing TF. We require the NMDFB to have PR property $[20,25]$. This condition translates to (1) zero aliasing TF $A\left(Z W_{D}^{d}\right) G$ $(Z)=0, \forall d=1, \ldots, D-1$ and (2) distortionless signal TF, $A(Z) \quad G(Z)=H^{\mathrm{NYQ}}(Z)$, where $H^{\mathrm{NYQ}}(Z)$ is any Nyquist pulse.

\subsection{The filtering property of PR-NMDFB}

The PR-NMDFB-based filtering is discussed intensively in [16]; here, we briefly review its concept and establish our notation. Given the discrete time Fourier transform (DTFT) of a target spectrum $S(\omega)$ and its finite time duration or truncated impulse responses $s(n), n=0,1, \ldots$ $N_{\text {max }}$, the goal is to synthesize or approximate it via an $M$-path PR-MNDFB.

Consider the NMDFB and time domain filtering model shown in Figure 3a,b. In Figure 3a, the input signal $r(n)$ is passed through an $M$-path, decimation by $D$, AFB/SFB with diagonal IPE matrix $\mathbb{K}_{M \times M}$, whose $(m \mathrm{M})$ th entry is a 


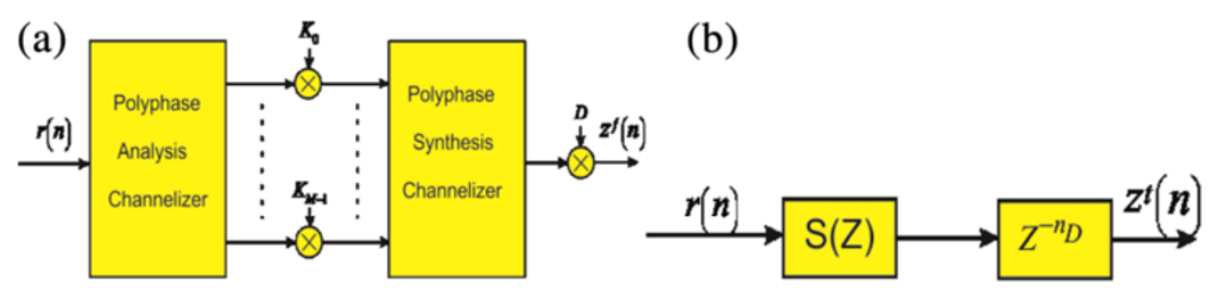

Figure 3 NMDFB domain filtering (a) and time domain filtering (b).

complex scalar denoted as $K_{m}$, for $m=0,1, \ldots, M-1$. In Figure $3 \mathrm{~b}$, the same signal $r(n)$ is fed into an FIR filter $S$ $(Z)=\mathcal{Z}\{s(n)\}$ and then delayed by $n_{D}$, where $n_{D}$ is the total delay introduced by the $M$-path, decimation by $D$, AFB/SFB. Using Equation 1, the Z-transform of the output signal $Z^{f}(n)$ corresponding to Figure $3 \mathrm{a}$ is written as $Z^{f}(Z)=T_{s}^{\mathbb{K}}(Z) R(Z)+\boldsymbol{T}_{A}^{\mathbb{K}}(Z) \overline{\boldsymbol{R}}(Z)$. As mentioned earlier, the aliasing energy can be made arbitrarily small by increasing AFB/SFB's stop-band attenuation. We now assume the aliasing energy is small and ignore it in the analysis. Therefore, we rewrite $Z^{f}(Z)$ as

$$
Z^{f}(Z)=T_{s}^{\mathbb{K}}(Z) R(Z)
$$

The $Z$-transform of the output signal $z^{t}(n)$ corresponding to the time domain filtering model shown in Figure $2 \mathrm{~b}$ can be written as

$$
Z^{t}(Z)=S(Z) Z^{-n_{D}} R(Z)
$$

The difference between the two filtering models can be defined as

$$
\mathcal{E}(Z)=\left[T_{s}^{\mathbb{K}}(Z) Z^{n_{D}}-S(Z)\right] R(Z) Z^{-n_{D}}
$$

Following Equation 4, we can further define the error TF to be

$$
\begin{aligned}
T_{\varepsilon}(Z) & =T_{s}^{\mathbb{K}}(Z) Z^{n_{D}}-S(Z) \\
& =\sum_{m=0}^{M-1} K_{m} H^{\mathrm{NYQ}}\left(Z W_{M}^{m}\right) Z^{n_{D}}-S(Z)
\end{aligned}
$$

Clearly, if the error TF $T_{\varepsilon}(Z)$ is zero or can be made arbitrarily small, then the NMDFB domain filtering becomes equivalent to the time domain filtering. Recall that $n_{D}$ is total delay of the Nyquist pulse $H^{\mathrm{NYQ}}$ $\left(Z W_{M}^{m}\right)$. Thus, the term $H^{\mathrm{NYQ}}\left(Z W_{M}^{m}\right) Z^{n_{D}}$ is the zerophase, non-causal version of the chosen Nyquist pulse that has a real spectrum. Let us denote the zero-phase Nyquist channel to be $\tilde{H}^{\mathrm{NYQ}}\left(Z W_{M}^{m}\right)$. We can further split Equation 5 into real and imaginary parts as shown in Equation 6:

$$
\begin{aligned}
T_{\varepsilon}(\omega)= & {\left[\sum_{m=0}^{M-1} \operatorname{Re}\left\{K_{m}\right\} \tilde{H}^{\mathrm{NYQ}}\left(\omega-\omega_{m}\right)-\operatorname{Re}\{S(\omega)\}\right] } \\
& +j\left[\sum_{m=0}^{M-1} \operatorname{Im}\left\{K_{m}\right\} \tilde{H}^{\mathrm{NYQ}}\left(\omega-\omega_{m}\right)-\operatorname{Im}\{S(\omega)\}\right]
\end{aligned}
$$

Equation 6 suggests the real and imaginary part of $K_{m}$ can be independently designed based on the real and imaginary part of the target spectrum $S(\omega)$. In addition, $\tilde{H}^{\mathrm{NYQ}}\left(\omega-\omega_{m}\right)$, is also a design parameter, since any waveforms satisfying the Nyquist channel condition can be used to design the analysis/synthesis LPPF. Note that the goal here is to approximate the target spectrum $S$ $(\omega)$. One option, as it is shown in [16], is to set $H^{\mathrm{NYQ}}(\omega)$ to be a gate function, i.e., sinc function in the time domain. This selection produces a piecewise constant approximation of the target spectrum $S(\omega)$, and Equations 5 and 6 can be viewed as the nearest neighbor interpolation error. Alternatively, setting $\tilde{H}^{\mathrm{NYQ}}(\omega)$ to be a triangularshaped Nyquist pulse, i.e. $\operatorname{sinc}^{2}$ in the time domain, produces a frequency domain linear interpolation between adjacent filter banks which means approximately a straight line interpolation of $S(\omega)$, and Equations 5 and 6 describe the linear interpolation error. In this case, the gain and phase distortion of the approximated spectrum are directly proportional to the second derivative of the target spectrum and inversely proportional to the squared analysis/synthesis path number $M$. In this paper, we shall adopt the triangular-shaped Nyquist pulse:

$$
\begin{aligned}
& \tilde{h}^{\mathrm{NYQ}}(n)=\frac{1}{M} \operatorname{sinc}^{2}\left(\frac{1}{M} n\right), \quad \text { for }-n_{d} \leq n \leq n_{d} \\
& \tilde{H}^{\mathrm{NYQ}}\left(\omega-\omega_{m}\right)=\left\{\begin{array}{c}
1-\left|\frac{\omega-\omega_{m}}{2 \pi / M}\right|, \omega \in\left[\begin{array}{c}
\left.\omega_{m}-\frac{2 \pi}{M}, \omega_{m}+\frac{2 \pi}{M}\right] \\
0
\end{array}, \quad\right. \text { otherwise }
\end{array}\right.
\end{aligned}
$$

In practice, as detailed in [16], this filter can be designed by using a windowed (i.e., Kaiser) sinc function.

One preliminary application of the filtering property in a FBSC receiver is to perform square root raised cosine 
(SRRC) shaping or matched filtering. In this case, the target spectrum $S(\omega)$ can be set equal to the ideal SRRC spectrum, and the IPE gains $K_{m}=S\left(\omega_{m}\right)$. Due to the underlying triangular pulse shape, the approximated spectrum by $K_{m}, K_{m}+1$ for $\omega \in\left[\omega_{m}, \omega_{m+1}\right]$, can be written as $\left\{\left(\omega_{m+1}-\omega\right) \cdot K_{m}+\omega \cdot K_{m+1}\right\}$. Using Equation 5 and only considering region $\omega \in\left[\omega_{m}, \omega_{m+1}\right]$, we can define the path-wise error TF on the interval $\omega \in$ $\left[\omega_{m}, \omega_{m+1}\right]$ as

$$
T_{\varepsilon, m}(\omega) \stackrel{\text { def }}{=}\left(\omega_{m+1}-\omega\right) \cdot K_{m}+\omega \cdot K_{m+1}-S(\omega)
$$

Set $K_{m}=S\left(\omega_{m}\right)$ and $K_{m+1}=S\left(\omega_{m+1}\right)$ and assume the target spectrum is analytic and twice differentiable, we can expand $S(\omega)$ using Taylor series around $=\omega_{m}$; we find

$$
S(\omega)=S\left(\omega_{m}\right)+\dot{S}\left(\omega_{m}\right)\left(\omega-\omega_{m}\right)+\frac{1}{2} \ddot{S}(\eta)\left(\omega-\omega_{m}\right)^{2}
$$

for some $\eta \in\left[\omega_{m}, \omega_{m+1}\right]$. Now, the maximum gain error within region $\left[\omega_{m}, \omega_{m+1}\right]$ can be bounded by

$$
\left|T_{\varepsilon, m}(\omega)\right| \leq \underset{\omega \in\left[\omega_{m}, \omega_{m+1}\right]}{\operatorname{Max}}|\ddot{S}(\omega)| \cdot \frac{1}{2}\left(\frac{\pi}{M}\right)^{2}=B_{\varepsilon, m}
$$

Let $\xi_{s, m} \stackrel{\text { def }}{=} \min _{\omega \in\left[\omega_{m}-\frac{\pi}{M}, \omega_{m} \pm \frac{\pi}{M}\right]}|S(\omega)|$; the maximum phase distortion $\phi_{m}$ can be readily obtained as

$$
\phi_{m} \leq \operatorname{atan}\left(\frac{B_{\varepsilon, m}}{\sqrt{\left(\xi_{s, m}\right)^{2}-\left(B_{\varepsilon, m}\right)^{2}}}\right) \text {, for } \xi_{s, m}>B_{\varepsilon, m}
$$

Note that, if $\gamma_{s, m} \leq B_{\varepsilon, m}$, the phase distortion is from $-\pi$ to $\pi$. However, this also implies the target spectrum $S(\omega)$ has significant attenuation at $\omega_{m}$ and the signal around frequency $\omega_{m}$ may not have significant value, i.e., in the stop-band of the target spectrum.

\section{PR-NMDFB-based carrier frequency recovery}

The conventional maximum likelihood carrier recovery approaches [26-28], as shown in Figure 4, requires implementing a pair of band-edge $(\mathrm{BE})$ filters that operate in parallel with the SRRC filter. The two BE filters are centered on the received signal's left and right transition bands. The carrier recovery is achieved by balancing the signal energy observed at the output of two BE filters. The offset correction process is achieved via a digital phase locked loop (PLL) and a direct digital synthesizer (DDS). As derived in [27], the optimal BE or frequency matched filter has frequency response equal to the frequency derivative of the SRRC MF, as shown in Figure 5a. Due to the difficulty of designing optimal BEs' sharp transition band, suboptimal BE filter is often used, as shown in Figure 5b, which makes BE filters have the

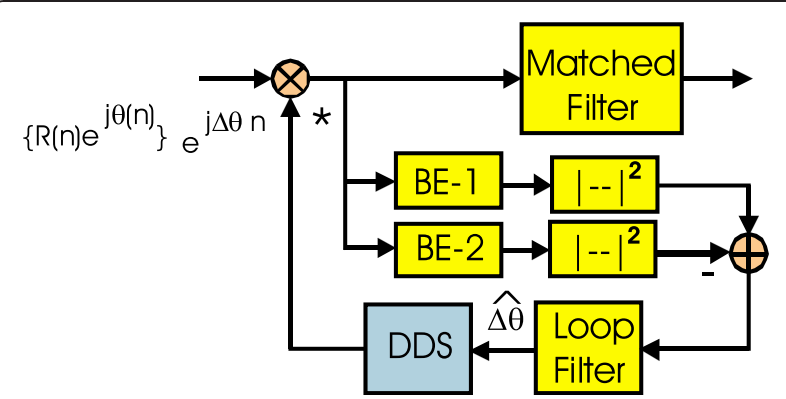

Figure 4 Band-edge filter frequency locked loop.

same length as the SRRC MF filter. As the signal's bandwidth grows, not only the power consumption of these three filters grows, but it also adds difficulty to lay out high-speed circuits running at the speed of many times the input rate.

Let us examine a baseband equivalent system for a QAM signal with complex notation for the in-phase $(I)$ and quadrature $(Q)$ branches, e.g., $\operatorname{Re}($.$) and \operatorname{Im}($.$) , re-$ spectively. Denote the $k$ th complex QAM data symbol as $S_{k}$, with the symbol period $T$ in seconds. Let the transmitter's SRRC shaping pulse be $h_{t x}(t)$ and additive white noise $n(t)=n_{I}(t)+j n_{Q}(t)$. Thus, the received and digitized signal with the sampling speed $T_{s}=\frac{1}{f_{s}}=T / 2$, i.e., two samples per symbol is written as

$$
r(n)=e^{j\left(\omega_{c} n+\psi\right)} \sum_{k=-\infty}^{\infty} S_{k} h_{t x}(n-2 k-\tau)+n(n)
$$

where $e^{j\left(\omega_{c} n+\psi\right)}$ is the residue CFO and phase offset after down-conversion and $\tau$ is the sampler time offset. Note, we do not consider the multipath channel effects in this paper. The equalization for FBSC is treated in [11]; it can be easily shown from PR-NMDFB filtering property that setting IPE as the linear equalizer response is equivalent to an FIR linear equalizer.

Figure 6 shows the proposed CFO recovery scheme. The CFO and constellation phase offset removal is achieved via dedicated IPEs embedded in between the receiver's PAC and PSC along with other filtering tasks such as matched filtering and equalization. The IPEbased CFO correction converts the high-speed time domain DDS into a collection of low-speed DDSs working in the channelizer-transformed domain. The BE filters can surely be implemented via the NMDFB filtering property along with the receiver's MF and equalizer. Moreover, since the BE filters [27] were oversampled (see Figure 5b) and only the BE output signal energy is needed, they can be synthesized with reduced sample rate via PSC with much fewer paths as depicted in Figure 6. The constellation phase offset removal is a 

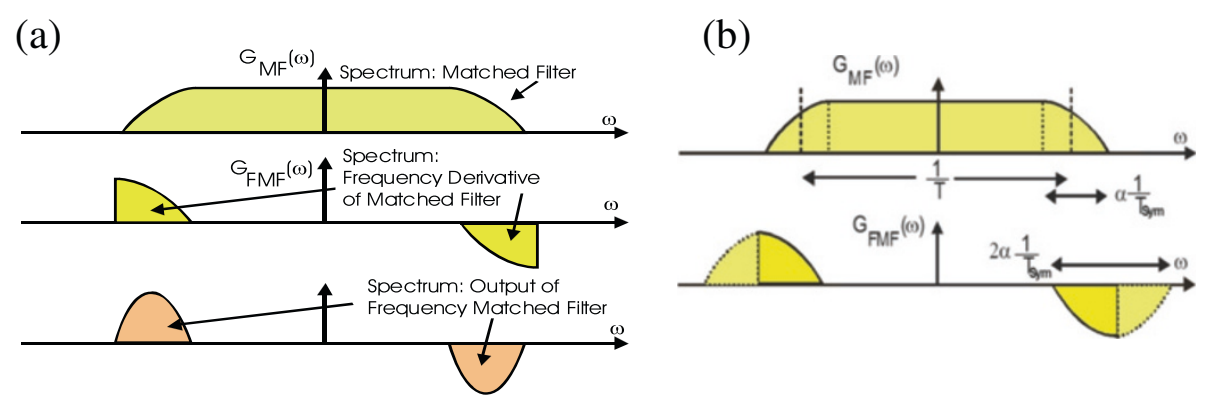

Figure 5 Spectra of SRRC MF, corresponding frequency MF, and spectral response of their cascade (a); suboptimal BE filters (b).

decision-directed process and happens after the CFO correction. It uses the same IPE as used in the CFO loop, and it is relatively simple to design. In this paper, we only focus on developing the BE filtering and CFO correction process; we will derive the operating conditions of IPE-based CFO correction, and we will show the design of PR-NMDFB-based BE filters.

\subsection{CFO correction in filter -bank-transformed domain}

To compensate the CFO, the signals presented at the output of PAC shall be de-rotated by $e^{-j \omega_{c} n D}$. Let us only consider the CFO correction IPE and ignore other IPEs for now, i.e., setting $\mathbb{K}_{M \times M}=I$. The $Z$-transform of the output signal $z(n)$ after frequency shift IPE is written as

$$
\begin{aligned}
Z(Z) & =\frac{1}{D} G_{1 \times M}^{T}(Z) \mathbb{K}_{M \times M} \mathbb{A}_{M \times D}\left(Z e^{j \omega_{c}}\right) \mathbf{R}_{D \times 1}\left(Z e^{j \omega_{c}}\right) \\
& =\frac{1}{D} T_{s}^{\mathbb{K}}(Z) R\left(Z e^{j \omega_{c}}\right)+\frac{1}{D} \boldsymbol{T}_{A}^{\mathbb{K}}(Z) \overline{\boldsymbol{R}}\left(Z e^{j \omega_{c}}\right)
\end{aligned}
$$

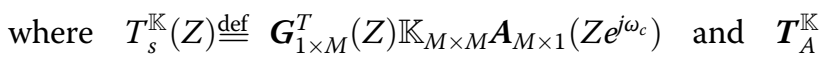
$(Z) \stackrel{\text { def }}{=} \boldsymbol{G}_{1 \times M}^{T}(Z) \mathbb{K}_{M \times M} \overline{\mathbb{A}}_{M \times(D-1)}\left(Z e^{j \omega_{c}}\right)$. The aliasing cancelation condition is verified if $\boldsymbol{T}_{A}^{\mathbb{K}}(Z)=0$, which can be translated to: $A\left(Z W_{D}^{d} e^{j \omega_{c}}\right) G(Z)=0, \forall d=1, \ldots, D$. It

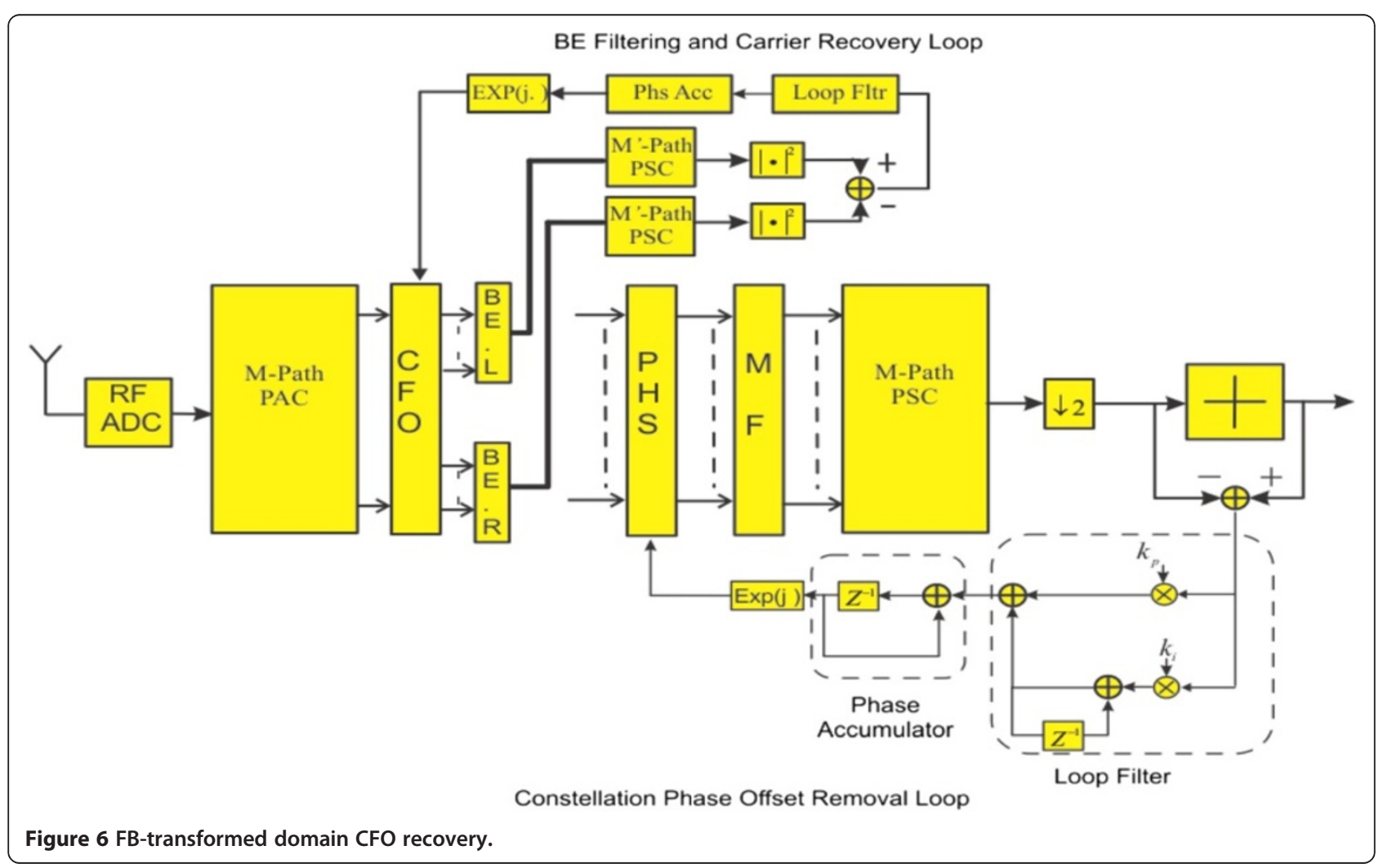


should be noted that the aliasing cancelation comes from the NMDFB prototype filter design and is unaffected by scalar diagonal matrix $\mathbb{K}$. The frequencytranslated PR condition is verified if $T_{s}^{\mathbb{K}}(Z)=\sum_{m=0}^{M-1} A$ $\left(Z W_{M}^{m} e^{j \omega_{c}}\right) G\left(Z W_{M}^{m}\right)=Z^{-n_{D}}$. This condition is met if the composite response $A\left(Z e^{j \omega_{c}}\right) G(Z)$ produces Nyquist pulse $H^{\mathrm{NYQ}}(Z)$ or its frequency-shifted version $H^{\mathrm{NYQ}}$ $\left(Z e^{j \omega_{c}}\right)$. We can design either $A(Z)$ or $G(Z)$ to be a Nyquist pulse and let the other filter be a low-pass filter that passes the Nyquist pulse unaltered. Equation 12 shows the impact of the two options, and we can see both choices produce the frequency-shifted version of the input signal when $\mathbb{K}_{M \times M}=I$ as along as the PR and aliasing cancelation conditions are met.

$$
\begin{aligned}
A\left(Z e^{j \omega_{c}}\right) G(Z) & =H^{\mathrm{NYQ}}\left(Z e^{j \omega_{c}}\right) \Rightarrow T_{s}^{\mathbb{K}}(Z) \\
& =\sum_{m=0}^{M-1} K_{m} H^{\mathrm{NYQ}}\left(Z W_{M}^{m} e^{j \omega_{c}}\right) \\
A\left(Z e^{j \omega_{c}}\right) G(Z) & =H^{\mathrm{NYQ}}(Z) \Rightarrow T_{s}^{\mathbb{K}}(Z) \\
& =\sum_{m=0}^{M-1} K_{m} H^{\mathrm{NYQ}}\left(Z W_{M}^{m}\right)
\end{aligned}
$$

Figure 7 depicts the example of the analysis and synthesis LPPF for an $M$-path, decimation by $D=M / 3$, PR-NMDFB that matches Equation 12b. The analysis LPPF is assigned to be a low-pass filter, while the synthesis LPPF is chosen to be a triangular-shaped Nyquist pulse. For $D=M / 3$ case, the PAC outputs have sample rate $\frac{3 \times f_{s}}{M}$ or range from $-\frac{3 \pi}{M}$ to $\frac{3 \pi}{M}$ given $f_{s}=2 \pi$. As seen from Figure 7, one can frequency shift the analysis LPPF without raising aliasing energy inside the 'aliasing free shift region.' However, the aliasing error will occur if we shift into the 'aliasing penalty shift region,' i.e., CFO $\omega_{c}$ approaches $\frac{\pi}{M}$. Clearly, this aliasing error is due to the transition band of the analysis LPPF as depicted in Figure 7. Note that a maximum frequency shift by $\frac{\pi}{M}$ is all we need to achieve full-range center frequency change, i.e., $\omega_{c} \in\left[-\frac{f_{s}}{2}, \frac{f_{s}}{2}\right]$ in PR-NMDFB-based FBMC receiver. This is because one can cyclic shift the PAC outputs to account for CFO greater than half of the NMDFB channel spacing, recall the path/channel spacing is $\frac{2 \pi}{M}$ in an $M$-path PR-NMDFB. Furthermore, it can be easily prove that the aliasing penalty shift region can be completely eliminated by having $D=M / 4$, yet reducing the decimation rate for this option also raises the system's workload.

\subsection{CFO detection in FB-transformed domain}

The CFO detection is based on a pair of BE filters centered on the left and right of the received signal's transition bands. They can be efficiently integrated in the NMDFB structure via spectral shaping IPEs. As shown in [27], the target spectrum of the two BE filters is the frequency derivative of the SRRC shaping filter's left and right transition bands. To synthesize an NMDFB-based BE filter, one simply sets the target spectrum $S(\omega)$ to be

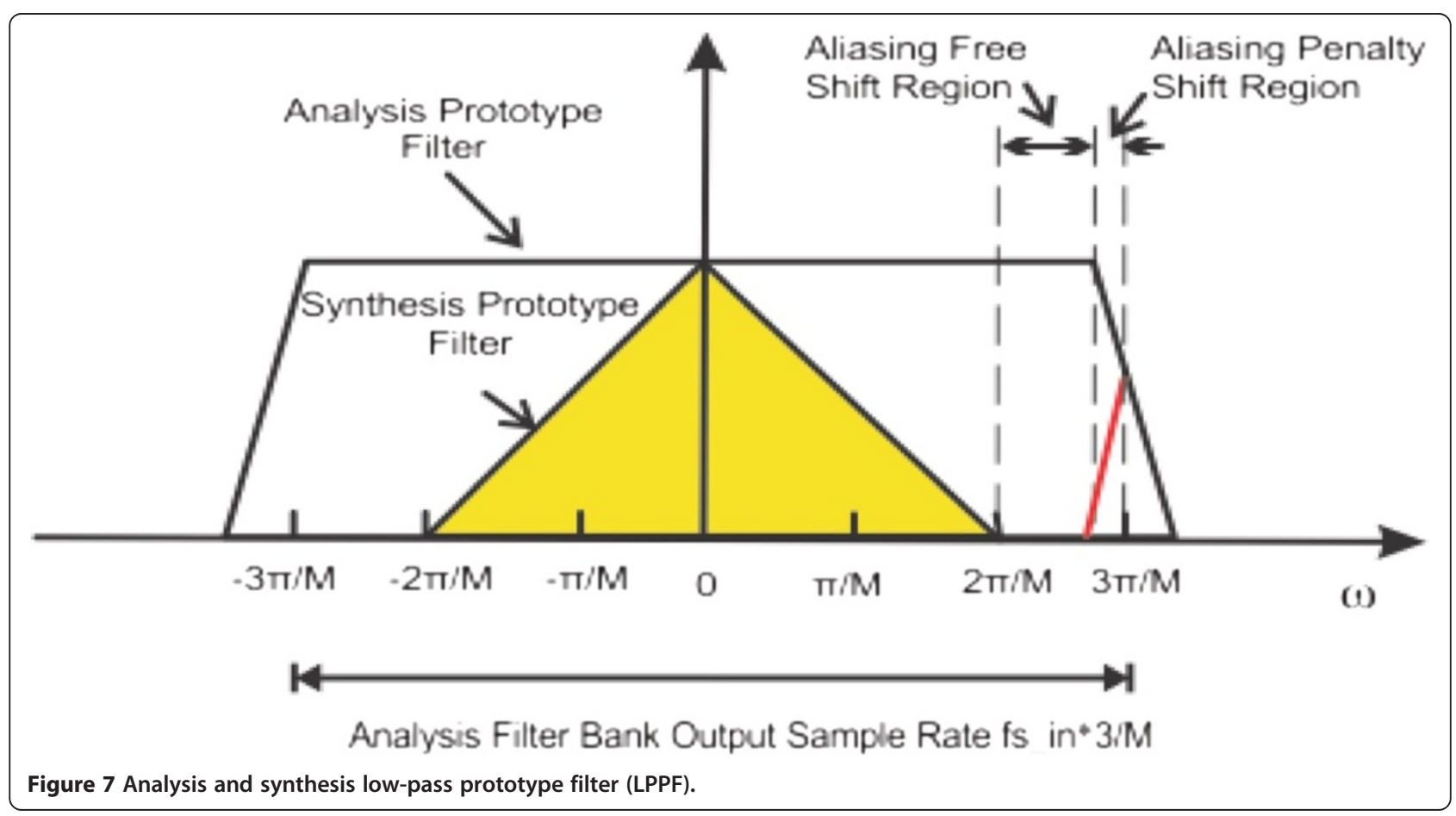


the ideal BE filter spectrum with IPE gain $K_{m}=S\left(\omega_{m}\right)$. Unlike the conventional time domain implementation (Figure 5b) in which suboptimal, symmetrically extended $\mathrm{BE}$ filters have to be built to avoid designing sharp filter transition bands, the PR-NMDFB-based filter allows us to directly synthesize the ideal $\mathrm{BE}$ filter spectrum and produce near-optimal frequency response as shown in Figure 5a. We will further demonstrate this in the simulation section.

Serving as the error detector within a PLL, the input signal to the BE filter must already be frequency-shifted or error-compensated as shown in Figure 4. Re-examine Equation 12 and consider a non-trivial IPE, i.e., $\mathbb{K}_{M \times M} \neq I$, Equation 12a corresponds to filter the input data and then deliver the heterodyned output, whereas Equation $12 \mathrm{~b}$ maps to first heterodyne the input signal and then apply the filtering. Clearly, the arrangement in Equation 12b matches the requirement of a loop control system. Therefore, the NMDFB implementation requires the analysis LPPF to be a low-pass filter and synthesis LPPF to be any Nyquist pulse, i.e., Figure 7. Another detail which is worth noting is that the conventional $\mathrm{BE}$ design produces an oversampled filter (see Figure 5). The oversampling ratio is related to the SRRC filter's transition bandwidth or the roll-off factor. Mapping to the NMDFB implementation, this means only a certain group of entries of BE filter's IPE matrix $\mathbb{K}_{M \times M}$ is non-zero. Equivalent of saying, only a portion of $\mathrm{FB}$ channels are needed to synthesize a $\mathrm{BE}$ filter. Therefore, $M^{\prime}$-path, reduced size PSC is used to only synthesize the FB channels that fall into the signal's left or right transition bands. This saves computational resources and increases the efficiency of the proposed architecture.

\section{PR-NMDFB-based symbol timing recovery}

The goal of a symbol timing synchronizer is to sample the MF output at optimum instant for the $k$ th symbol. The conventional analog solution controls a sampleand-hold circuit located at the output of the analog MF to identify the optimum sampling instant. It is also well known that $[29,30]$ the $\log$-likelihood function for unknown timing phase $\tau$ for equally likely $S_{k} \in\{-1,+1\}$ is

$$
\Lambda(\tau)=\sum_{k} \ln \left[\left(\cosh \left(\frac{2 E}{N_{0}}\right) y(k T+\tau)\right)\right]
$$

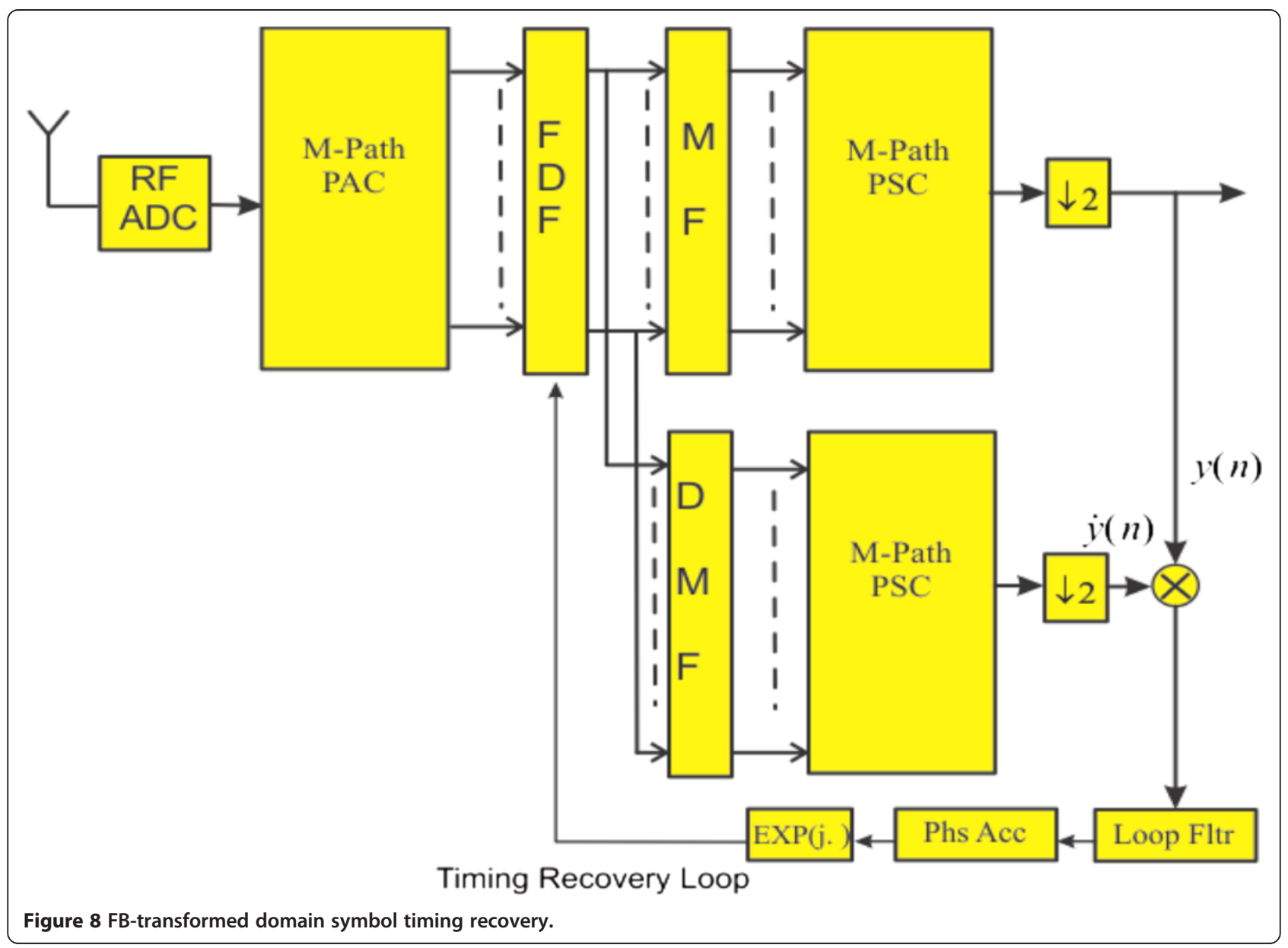


where $E$ is the symbol energy, $N_{0}$ is the power spectral density of zero-mean Gaussian noise, and $y(k T+\tau)$ is the analog MF output. The estimate $\hat{\tau}$ that maximizes $\Lambda(\tau)$ is the timing phase that forces the derivative of $\Lambda(\tau)$ to zero.

$$
\sum_{k} \tanh \left(\frac{2 E}{N_{0}} y(k T+\tau)\right) \frac{d}{d t} y(k T+\tau)=0
$$

Often, the $\tanh ($.$) function is replaced by its small sig-$ nal approximation $\tanh (x) \approx x$ for low signal-to-noise ratio (SNR) and $\tanh (x) \approx \operatorname{sign}(x)$ for high SNR. The authors in [29] proposed full digital timing recovery techniques based on polyphase FB and have shown various timing error detection (TED) methods via FBs. In this paper, we adopt the TED formed by taking the product between the MF output and the derivative MF output, i.e., Figure ten of [29]. The derivative MF is produced by taking the derivative to the time domain SRRC impulse response; this should not be confused with $\mathrm{BE}$ filter which is formed by taking the frequency domain derivative of the SRRC spectra.

\subsection{Timing error correction in FB-transformed domain}

The digital timing error correction requires building a fractional delay filter (FDF). The FDF is conventionally implemented in the time domain either via Farrow structure [31] which is a polynomial-based interpolator or via polyphase interpolators [26]. In this section, we introduce the NMDFB-based FDF.

The time domain perspective has led us to build interpolators acting on the desired time grid between available samples. Notice that the basic Fourier transform property states that time delay is phase shift in the frequency domain, and the phase shifter has frequency response $S(\Omega)=e^{j \Omega \tau}$, where $\Omega$ is the continuous frequency in Fourier transform. Using $K_{m}=S\left(\omega_{m}\right)$, for $\omega_{m}=\frac{2 \pi}{M} m$, $m=0,1, \ldots, M-1$, gives $K_{m}=e^{j \frac{2 \pi}{M} m \tau},|\tau| \leq 0.5, m \neq \frac{M}{2}$. Note that the DTFT must have a periodic spectrum. Therefore, we force $K_{M / 2}$, the channel located on the half sample rate, to be the average of its adjacent neighbors:

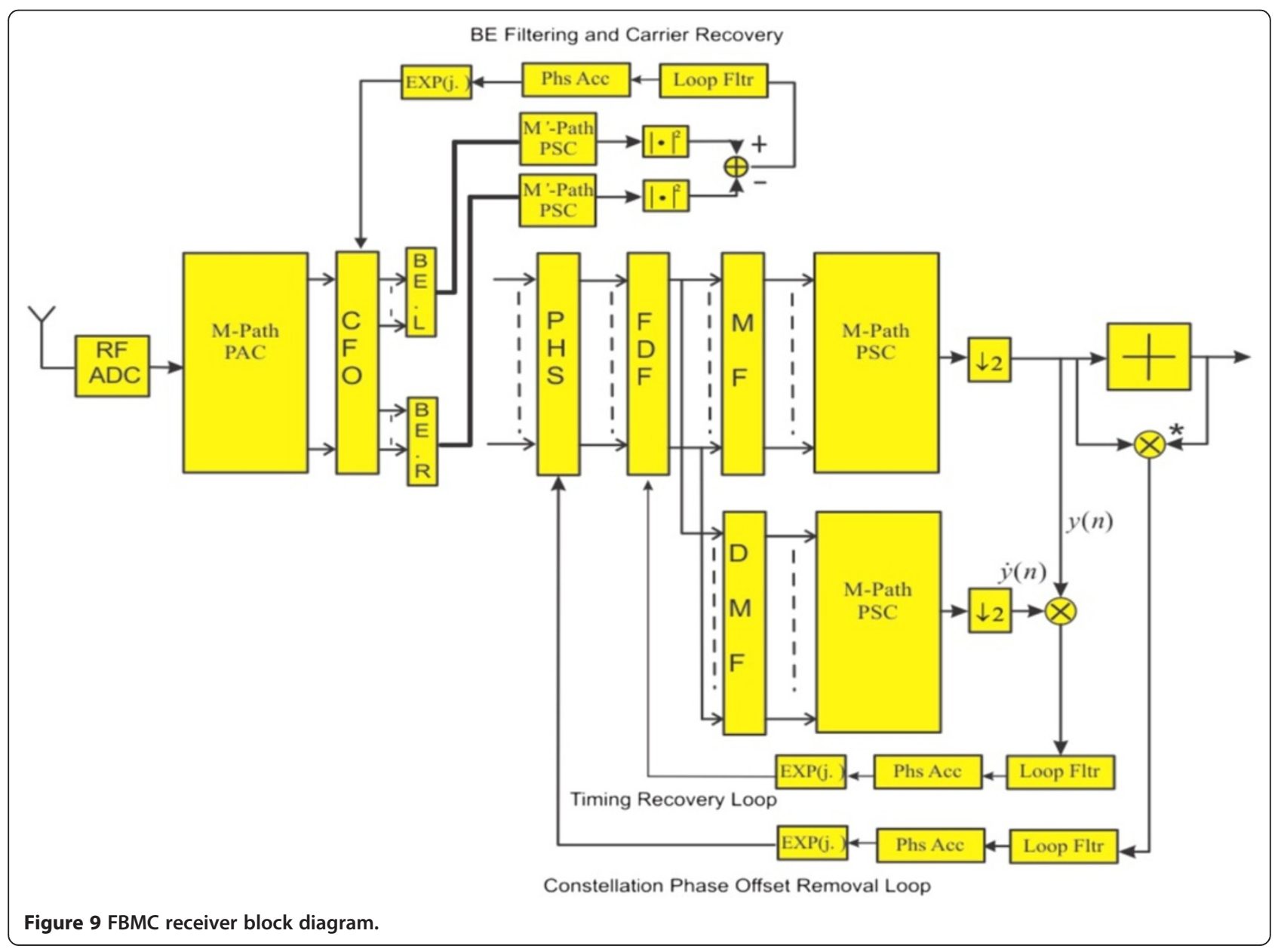


$K_{M / 2}=\frac{1}{2}\left(K_{M / 2-1}+K_{M / 2+1}\right)$. Doing so, we made the $K_{m}, m=0, \ldots, M-1$ periodic, but we also sacrificed the half sample rate channel. The operation bandwidth for this fractional delay filter becomes $\omega \in\left[-\pi+\frac{\pi}{M}, \pi-\frac{\pi}{M}\right]$.

$$
K_{m}=\left\{\begin{array}{c}
e^{j \frac{2 \pi}{M} m \tau}, f \text { or } m \neq \frac{M}{2} \\
\frac{1}{2}\left(K_{M / 2-1}+K_{M / 2+1}\right), f \text { or } m=\frac{M}{2}
\end{array}\right.
$$

From the spectral approximation point of view, the problem becomes using $M$ spectral samples to approximate complex sinusoid $e^{j \frac{2 \pi}{M} m \tau}$ up to half a cycle. In the case of straight line approximation and $M=64$, we use Equations 7 to 9 to determine the maximum magnitude error to be $-70 \mathrm{~dB}$ and maximum phase distortion to be $3.012 \times 10^{-4} \mathrm{rad}$. It is clear that the spectral approximation provides a very good FDF with only 64-path filter banks.

\subsection{Timing error detection in FB-transformed domain} The time domain TED, Figure 10 of [29], requires building one FIR SRRC MF and one time domain derivative SRRC MF. The product of the two filters forms the TED which then drives a PLL. The PLL then tunes the FDF to find the best symbol timing. The NMDFB implementation of the TED together with the FDF is shown in Figure 8, where the IPEs are used to synthesize the FDF, MF, and the derivative MF. Their target spectrums can be readily obtained from the previously developed NMDFB filtering property in Section 2. As seen from Figure 8, one PAC and two PSCs are used to produce the time domain MF, derivative MF, as well as the FDF. We shall show a detailed workload analysis in the next section.

\section{Full NMDFB-based FBMC receiver and complexity analysis}

The complete block diagram of the FBMC receiver is shown in Figure 9. We can see the entire signal processing is embedded between the PAC- and PSC-transformed domain, and all filtering tasks are implemented based on the NMDFB filtering property via dedicated IPEs. In this section, we would like to analyze the workload for the FBMC receiver and compare it to its time domain antecedent counterpart.

Examining Figure 9, the FBSC receiver contains one $M$ path PAC, two $M$-path PSC, two reduced size $M^{\prime}$-path PSC, five $M$-entry IPEs, and two reduced size $M^{\prime}$-entry IPEs. Let all LPPFs have $L$ taps (real coefficient) per polyphase arm, i.e., the LPPF length for $M$-path PAC (or $M$ path PSC) is $M \times L$ and the LPPF length for $M^{\prime}$-path PSC is $M^{\prime} \times L$. Take the $M$-path, decimation by $D, P A C$ for example: a block of $D$ pieces of data enter the PAC, and its LPPF operates once, and so does its $M$-point FFT. Therefore, the workload in terms of the number of multiplies per input complex ( $I-Q$ pair) sample, for an $M$-path, decimation by $D, P A C$ is calculated as $\frac{1}{D}\left[2 M L+\left\langle M P n t^{\circ} F F T\right\rangle\right]$, where $\left\langle M P n t^{\circ} F F T\right\rangle$ denotes the number of multiplies required for an $M$-point FFT processing complex inputs. In addition, the workload for an $M$-entry complex coefficient IPE is $\frac{4 M}{D}$.

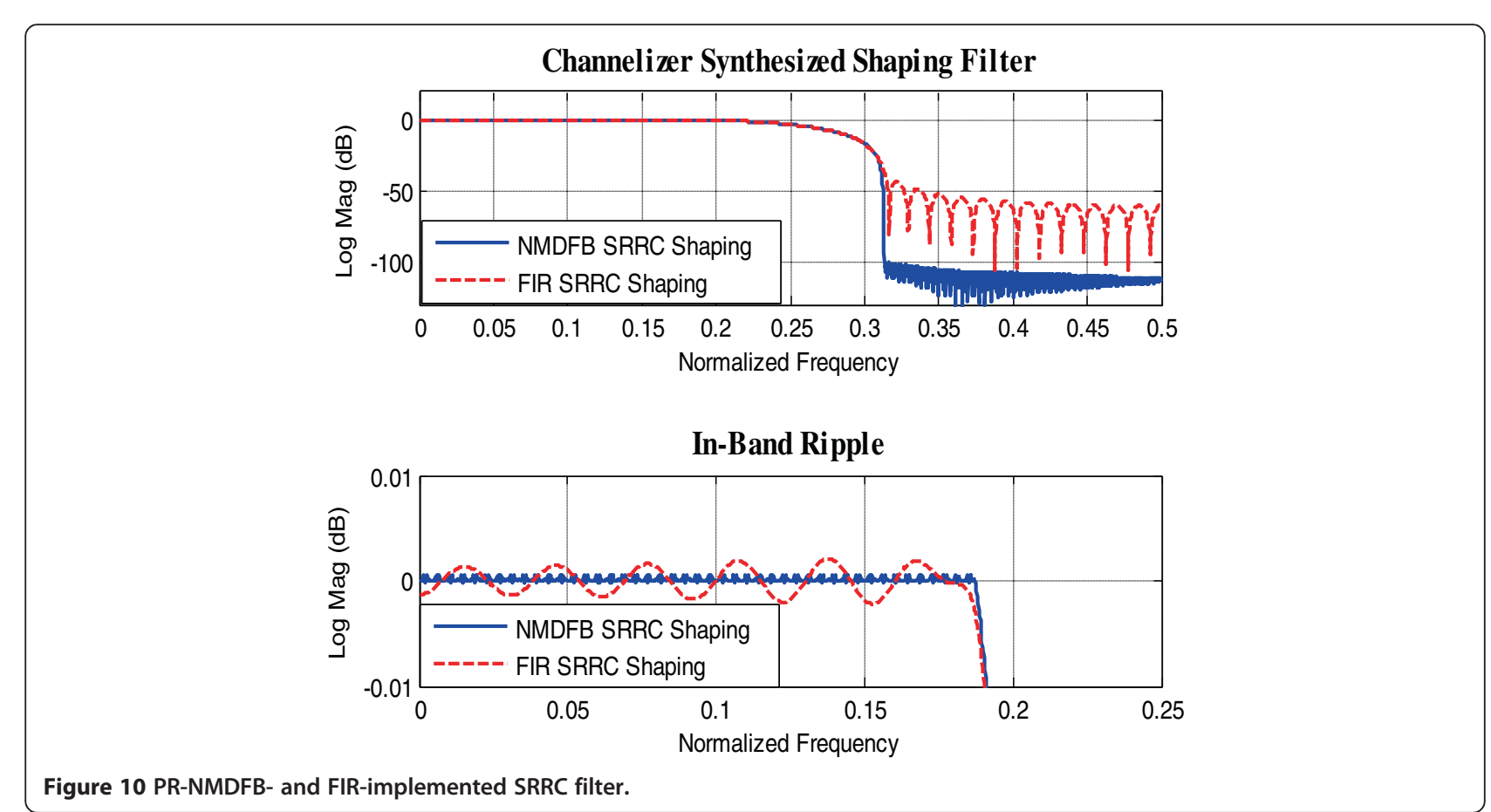




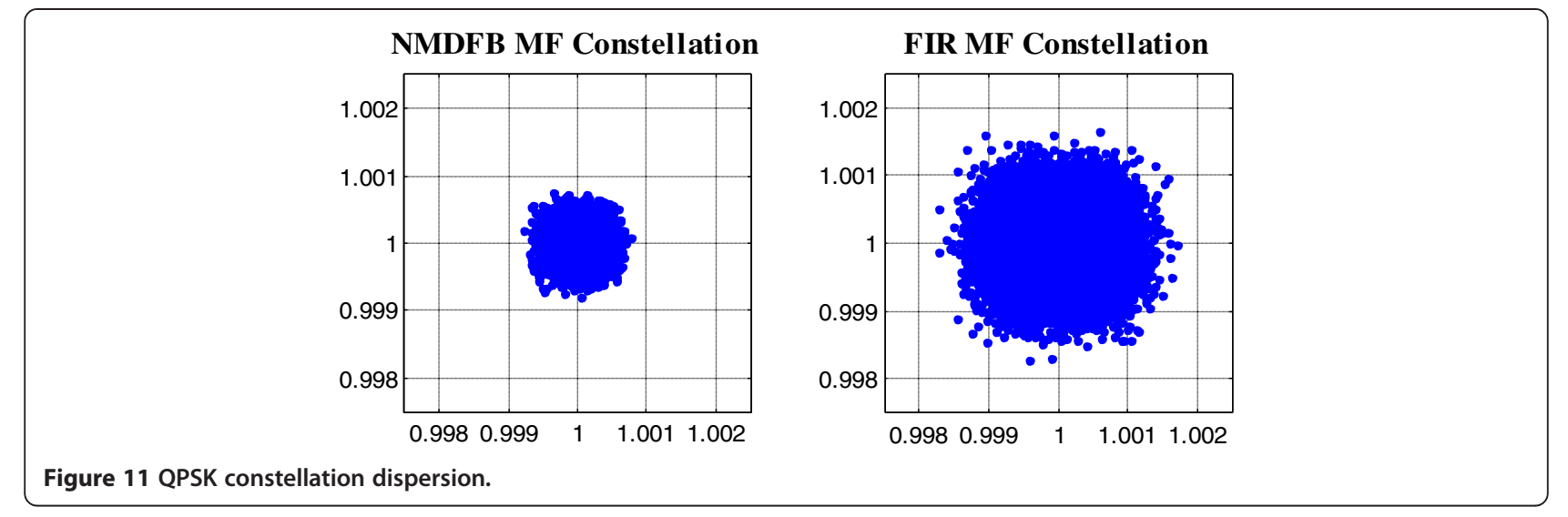

For an $M$-path, decimation by $D$ arrangement, every block of $D$ pieces of data coming in all the PACs, PSCs, and IPEs operate once. Therefore, the total workload count in terms of number of multiplies per input complex sample is

$$
\begin{aligned}
(\text { ops })_{\text {input }}= & \frac{1}{D}\left[6 M L+4 M^{\prime} L+20 M+8 M^{\prime}\right. \\
& \left.+3\left\langle M P n t^{\circ} F F T\right\rangle+2\left\langle M^{\prime} P n t^{\circ} F F T\right\rangle\right]
\end{aligned}
$$

Equation 14 is the total workload count for the proposed FBSC receiver. It should be noted that Equation 14 also includes the fractionally spaced equalizer, which is essentially another IPE that can be combined with the
MF IPE [16]. The conventional time domain implementation of Figure 8 requires building at least four FIR filters: SRRC MF, two BE filters (for carrier recovery), and one derivative MF (for timing recovery) [29]. These four filters often have the same length (suboptimal BE filter designed as Figure 5b) with the SRRC filter. Therefore, the workload count for time domain implementation is

$$
(\text { ops })_{\text {input }}=8 N
$$

where $N$ is the number of taps of SRRC filter.

We now assign practical parameters to Equations 14 and 15 to make further comparison. A 65-tap, i.e., $N=$ 64 , SRRC filter with $25 \%$ roll-off factor is used for time

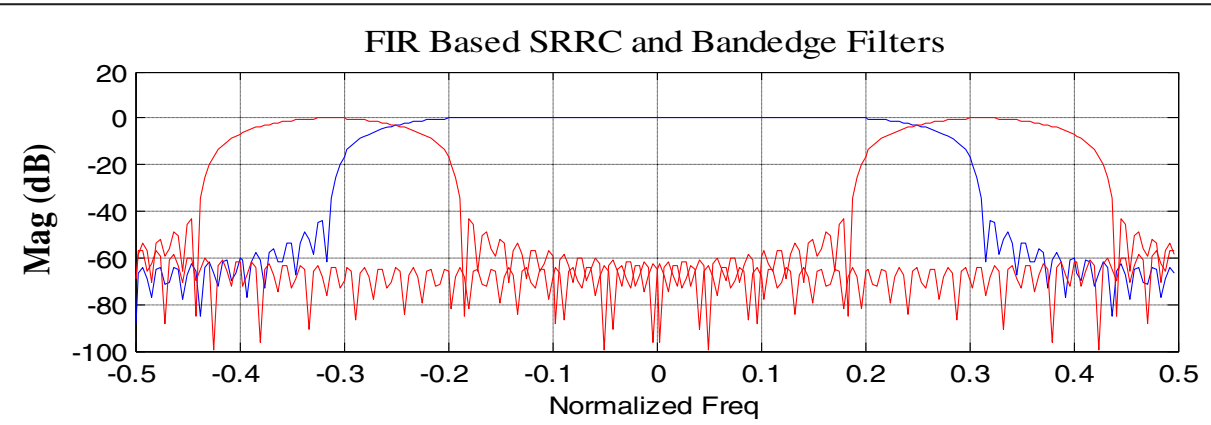

PR-NMDFB Based SRRC and Band Edge Filters

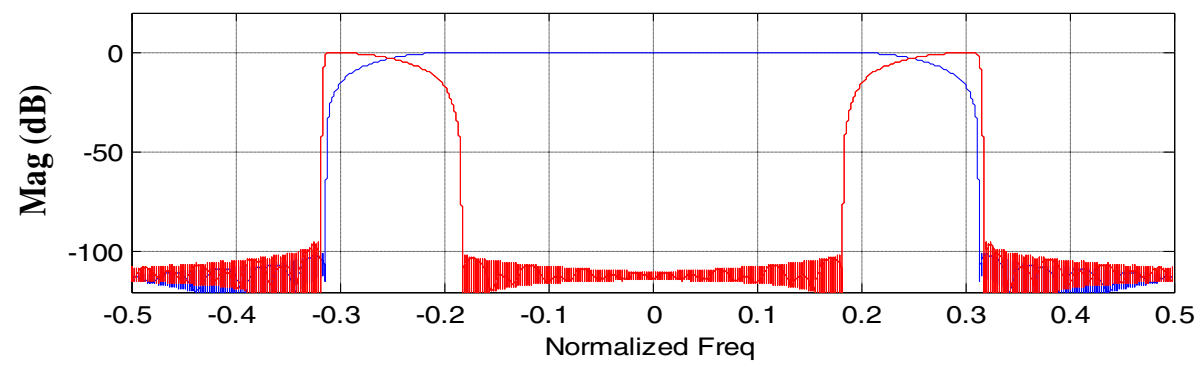

Figure 12 Time and channelizer domain MF and BE filters. 

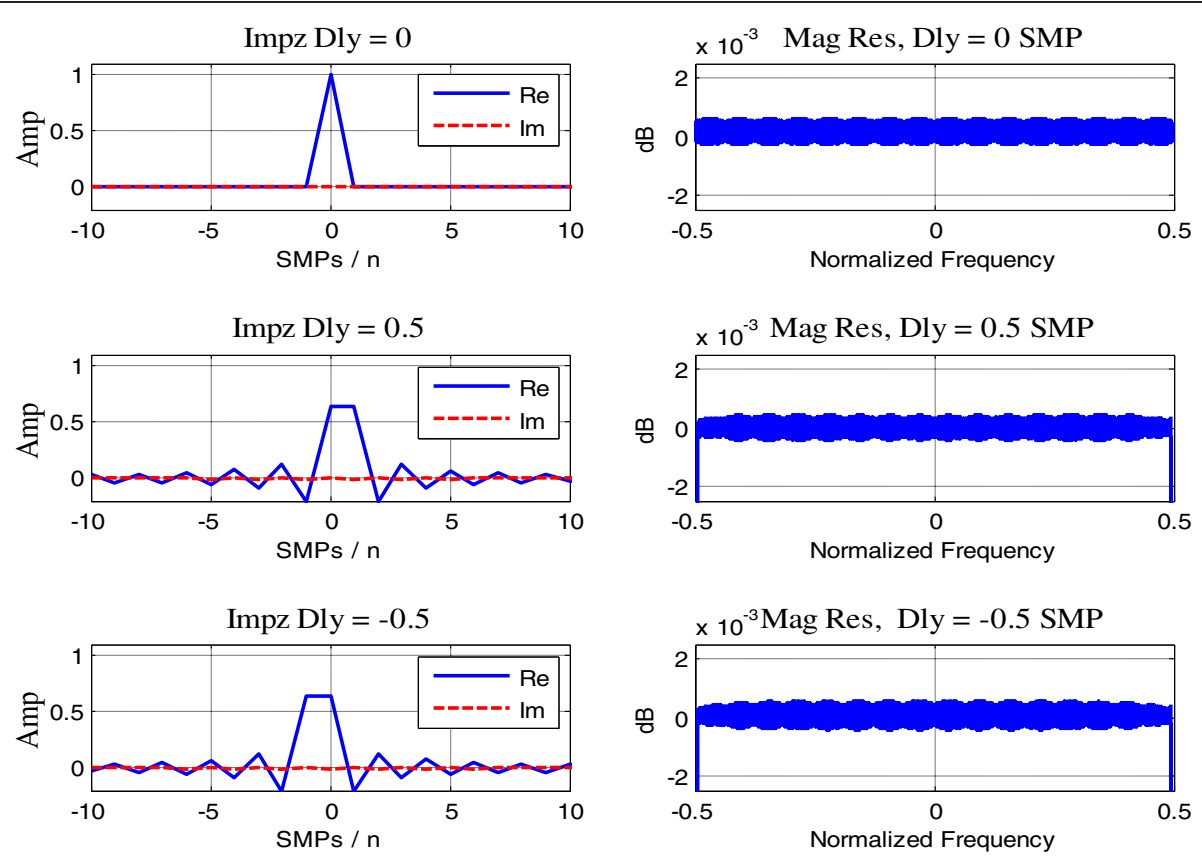

Figure $13 M=240, D=80$, PR-NMDFB-implemented FDF.

domain implementation. Therefore, based on Equation 15, the conventional FIR approach costs 520 real multiplies per complex input. In the FBSC solution, we use $M=240$, $M^{\prime}=42$, and $D=\frac{M}{3}=80$, triangular-shaped PR-NMDFB with LPPF length $L=8$, and our observation shows it can well equalize multipath channels with normalized RMS delay spread $\tau_{\mathrm{rms}} / T=5$. The 240-point and 42-point complex FFT cost 1,100 and 152 real multiplies, respectively, [32]. Plugging in those parameters into Equation 14, we find the workload count for FBSC receiver is 270 real multiplies per complex input. Those numbers show the PR-NMDFB-based FBSC solution offers 48\% workload

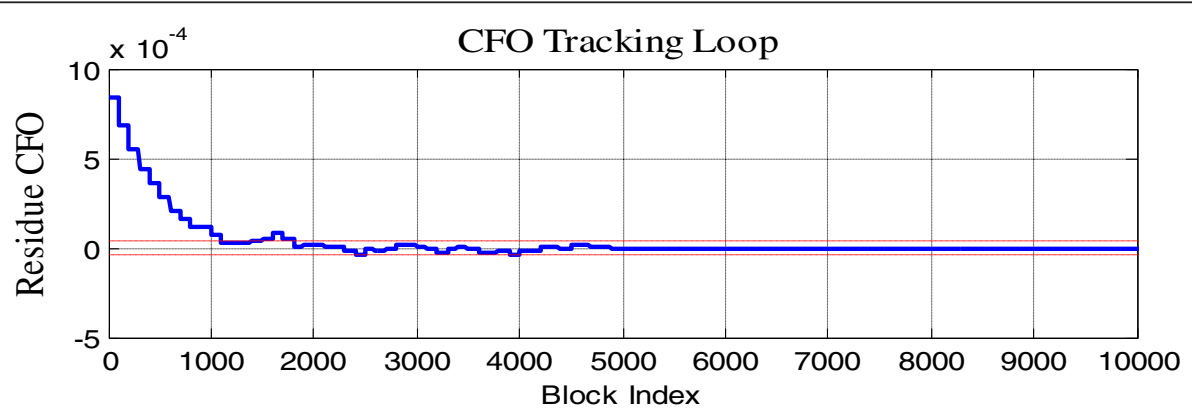

Energy Different at Band Edge Filters

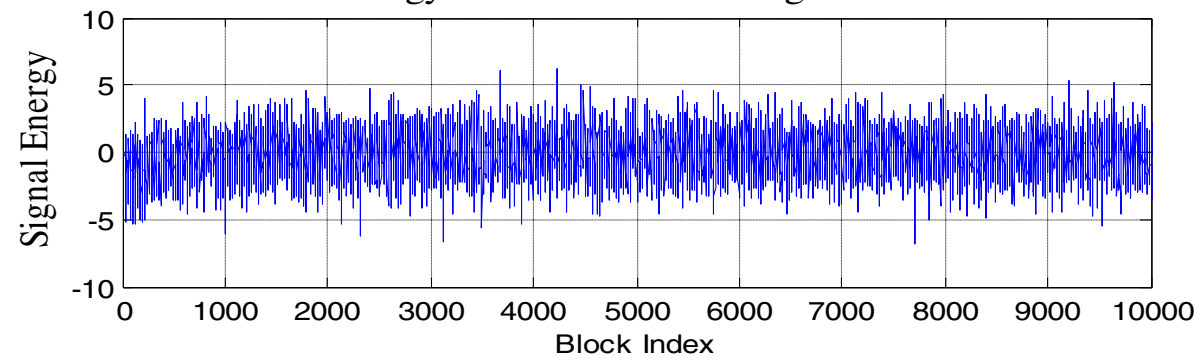

Figure 14 CFO tracking loop profile. 


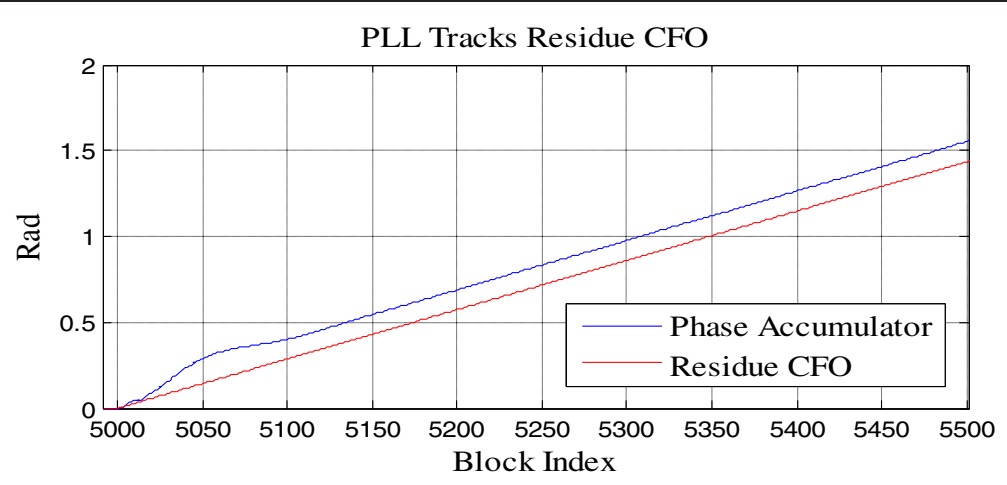

Figure 15 Constellation phase offset removal tracking loop profile.

reduction only for the synchronization part, and this workload reduction did not account for the linear equalization which is required in the conventional FIR approach; notice that, on the other hand, the equalization task has already been included in Equation 14. Furthermore, as will be shown in the simulation section, the 65-tap SRRC only gives 43-dB stop-band performance; however, the PR-NMDFB offers 100-dB dynamic range, an improvement by $56 \mathrm{~dB}$.

\section{Simulation results}

This section presents the simulation results of the proposed FBSC receiver. We shall first examine the PRNMDFB-implemented filters and compare them with the FIR-based designs. Then, we will demonstrate the behaviors of the proposed CFO recovery loop and symbol timing recovery loop.

\subsection{PR-NMDFB-implemented filters}

Figure 10 shows the magnitude response of the $M=240$, $D=80$, triangular-shaped PR-NMDFB-synthesized SRRC filter with $25 \%$ roll-off factor overlaid with 65-tap FIR SRRC filter. It can be shown that the power consumption of the two approaches is about the same (i.e., using PRNMDFB as SRRC filter only); however, their performance differs vastly. The PR-NMDFB-synthesized SRRC has over $50 \mathrm{~dB}$ advantage in stop-band performance with much smaller in-band ripple. Figure 11 shows the SRRC shaped and MF-ed QPSK constellation on the first quadrant without multipath channel and noise. The measured error vector magnitude (EVM) [33] for PR-NMDFB-based approach is $-73.02 \mathrm{~dB}$ or $0.022 \%$, and for FIR-based approach is $-65.95 \mathrm{~dB}$ or $0.050 \%$.

Figure 12 shows the FIR-based time domain implementation of the suboptimal 65-tap BE filters and the PRNMDFB-based BE filters. Comparing the two, we find the PR-NMDFB implements extremely sharp filter transitions and achieve near-optimal BE filter design [27]. Note that the smallest transition band of a triangular-shaped PRNMDFB is $\frac{\pi}{M}$, half of the NMDFB channel spacing. Again, the PR-NMDFB approach gives over $50 \mathrm{~dB}$ in stop-band performance compared to FIR-based BE filter design.

Figure 13 shows the result for delaying the impulse response by $0,0.5$ and -0.5 samples. The left column figures show the impulse responses, while the right column figures show the magnitude responses. Note that we have subtracted the parasitic delay $n_{D}$ caused by PAC/ PSC from the impulse responses. Thus, we see the impulse response for delaying by 0 sample sits on index 0 . The impulses for delaying by \pm 0.5 samples become sampled time offset Nyquist pulses. The magnitude responses for delaying by \pm 0.5 samples are seen to have very slightly larger in-band distortions comparing to delaying by 0 sample. This is due to the use of IPE to approximate the complex sinusoid of the phase shifters (see Equation 13). Clearly, the worst case distortion happens when delaying by \pm 0.5 , since all one need is fractional delay within one sample.

\subsection{CFO recovery results}

The CFO and constellation phase offset removal is simulated based on QPSK signal with $25-\mathrm{dB} E_{\mathrm{b}} / N_{0}$. The CFO is set to $\omega_{c}=0.2 \frac{2 \pi}{M}$ or $20 \%$ of the NMDFB channel
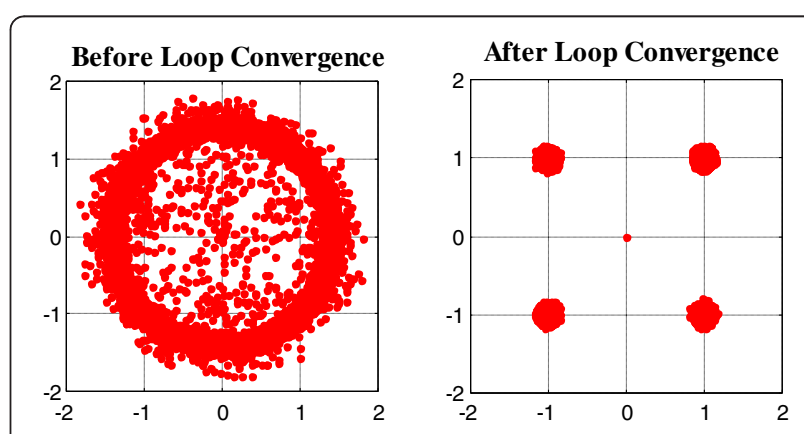

Figure 16 Constellation before and after the convergence of the constellation phase offset removal loop. 
PHASE ACCUMULATOR TIME PROFILE
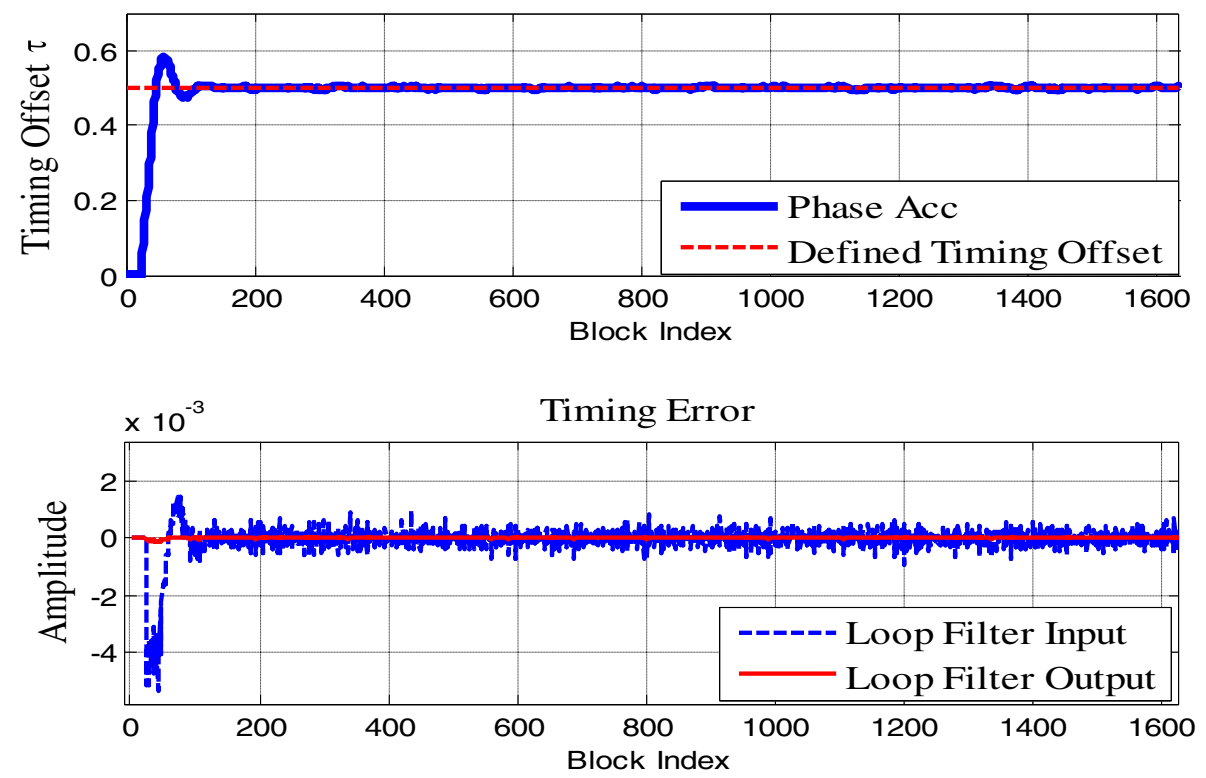

Figure 17 Symbol timing recovery loop profile.

spacing. Figure 14 shows the CFO tracking loop dynamics. The horizontal axis is plotted in terms of block index, recall the NMDFB receiver takes in data samples in block of $D$, and in this example $D=80$. We can see the PLL pulls the CFO to a very small value by balancing the signal energy from the two BE filters. Upon the convergence of the CFO loop, the constellation phase offset correction loop (Figure 15) began to work, which tracks the small residue CFO based on decision-directed criterion. The signal constellations taken before and after the convergence of the phase offset correction loop are shown in Figure 16.

\subsection{Symbol timing recovery results}

The symbol timing recovery is simulated under the same setup as the carrier recovery. The initial timing offset $\tau$ is set to be $0.5 T_{s}$. Figure 17 shows the phase accumulator profile of the timing recovery PLL, and we can see the steady state is reached in about 100 blocks or 8,000 samples. The lower subplot of Figure 17 shows the input and output signals at the loop filter, and we see the timing error is driven to zero by the PLL. Figure 18 shows the QPSK constellation before/after the convergence of timing loop, which further confirms the functionality of the proposed approach.

\section{Conclusion}

We have proposed the complete receiver structure for FBSC transmission with emphasis on receiver filters and synchronization. We applied the PR-NMDFB-based filtering property in designing the wideband carrier and symbol timing synchronization. This allows us to concurrently
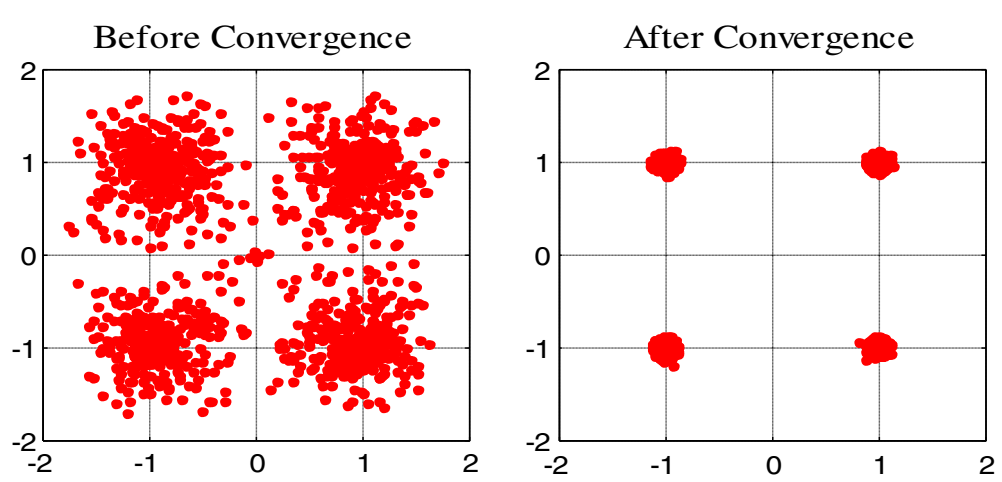

Figure 18 Constellation before and after the convergence of the symbol timing recovery loop. 
design $\mathrm{BE}$ filters and fractional delay filters within the entire FB-based receiver structure. The complexity analysis shows the proposed approach offers $48 \%$ workload reduction when only the synchronization tasks are considered, and further reduction in computation is expected if equalization is considered. Moreover, in contrast to the conventional FIR approach, which requires the hardware clock to run on many times of the input sample rate, the proposed FBSC receiver allows all the filtering tasks to operate on deeply decimated sample rate, which is a highly desired characteristic for the hardware implementation of fully digital receivers.

\section{Competing interests}

The authors declare that they have no competing interests.

\section{Author details}

${ }^{1}$ Department of Electrical and Computer Engineering, San Diego State University, San Diego, CA 92182-1309, USA. ${ }^{2}$ University of California at San Diego, La Jolla, CA 92093-0407, USA.

\section{Received: 22 November 2013 Accepted: 17 March 2014}

Published: 8 May 2014

\section{References}

1. S Ariyavisitakul, D Falconer, F Adachi, H Sari, Guest editorial - wireless broadband techniques. IEEE J. Select. Areas Commun. 17(10), 1709-1710 (1999)

2. VN Richard, R Prasad, OFDM Wireless Multimedia Communication (Artech House, Boston, 2000)

3. N Benvenuto, R Dinis, D Falconer, S Tomasin, Single carrier modulation with nonlinear frequency domain equalization: an idea whose time has come-again. Proc. IEEE 98(1), 69-96 (2010)

4. S Tomasin, N Benvenuto, Iterative design and detection of a DFE in the frequency domain. IEEE Trans. Commun. 53(11), 1867-1875 (2005)

5. G Huang, A Nix, S Armour, Feedback reliability calculation for an iterative block decision feedback equalizer, in 2009 IEEE 70th Vehicular Technology Conference Fall (VTC 2009-Fall) (Piscataway, IEEE, 2009), pp. 1-5

6. M Magarini, L Barletta, A Spalvieri, Efficient computation of the feedback filter for the hybrid decision feedback equalizer in highly dispersive channels. IEEE Trans. Wireless Commun. 11(6), 2245-2253 (2012)

7. C Zhang, Z Wang, C Pan, S Chen, L Hanzo, Low-complexity iterative frequency domain decision feedback equalization. Vehicular Technol. IEEE Trans. 60(3), 1295-1301 (2011)

8. T Ihalainen, A Ikhlef, J Louveaux, M Renfors, Channel equalization for multi-antenna FBMC/OQAM receivers. IEEE Trans. Veh. Technol. 60(5), 2070-2085 (2011)

9. M Caus, Al Perez-Neira, Transmitter-receiver designs for highly frequency selective channels in MIMO FBMC systems. Trans. Sig. Proc. 60(12), 6519-6532 (2012)

10. T Ihalainen, TH Stitz, M Rinne, M Renfors, Channel equalization in filter bank based multicarrier modulation for wireless communications. EURASIP Appl. Signal Process 2007, 140-140 (2007)

11. Y Yang, T Ihalainen, M Rinne, M Renfors, Frequency-domain equalization in single-carrier transmission: filter bank approach. EURASIP J. Adv. Signal Process 2007, 1-6 (2007)

12. S Weiss, SR Dooley, RW Stewart, AK Nandi, Adaptive equalization in oversampled subbands. Electron Lett. 34(15), 1452-1453 (1998)

13. Y Yang, TH Stitz, M Rinne, M Renfors, Mitigation of narrowband interference in SC transmission with filter bank equalization, in IEEE Asia Pacific Conference on Circuits and Systems, 2006. APCCAS 2006 (IEEE, Piscataway, 2006), pp. 748-751

14. Y Yang, $T$ Ihalainen, M Renfors, M Rinne, Noise predictive turbo equalization for a filter bank based receiver in a SC transmission system, in IEEE 65th Vehicular Technology Conference, 2007. VTC2007-Spring (IEEE, Piscataway, 2007), pp. 2389-2393

15. B Farhang-Boroujeny, Adaptive Filters: Theory and Applications (Wiley, Chichester, 1998)
16. X Chen, FJ Harris, E Venosa, BD Rao, Non-maximally decimated analysis/ synthesis filter banks: applications in wideband digital filtering. IEEE Trans. Signal Process. 62(4), 852-867 (2014). doi: 10.1109/TSP.2013.2295549

17. H Bolcskei, F Hlawatsch, Oversampled cosine modulated filter banks with perfect reconstruction. IEEE Trans. Circuits and Systems I| 45(8), 1057-1071 (1998)

18. Z Cvelkovic, M Vetterli, Oversampled filter banks. IEEE Trans. Signal Process 46(5), 1245-1255 (1998)

19. H Bolcskei, F Hlawatsch, HG Feichtinger, Frame-theoretic analysis of oversampled filter banks. IEEE Trans. Signal Process 46(12), 3256-3268 (1998)

20. T Karp, NJ Fliege, Modified DFT filter banks with perfect reconstruction. IEEE Trans. Circuits and Systems II 46(11), 1404-1414 (1999)

21. JM de Haan, Filter bank design for subband adaptive filtering (PhD dissertation. Department of Telecommunications and Signal Processing, University of Karlskrona and Ronneby, Sweden, 2001)

22. FJ Harris, X Chen, E Venosa, BD Rao, Wideband 160-channel polyphase filter bank cable TV channeliser. Signal Proc. IET 5(3), 325-332 (2011)

23. FJ Harris, E Venosa, X Chen, BD Rao, Polyphase analysis filter bank down-converts unequal channel bandwidths with arbitrary center frequencies. Analog Integr. Circ. S. 71(3), 481-494 (2012)

24. T Nguyen, Near-perfect-reconstruction pseudo-QMF banks. IEEE Trans. Signal Process 42(1), 65-76 (1994)

25. NJ Fliege, Multirate Digital Signal Processing (Wiley, Chichester, 1994)

26. FJ Harris, Multirate Signal Processing for Communication Systems (Prentice Hall, Upper Saddle River, 2004)

27. FJ Harris, E Venosa, X Chen, C Dick, Band edge filters perform non data-aided carrier and timing synchronization of software defined radio QAM receivers, in 2012 15th International Symposium on Wireless Personal Multimedia Communications (WPMC) (IEEE, Piscataway, 2012), pp. 271-275

28. C Dick, FJ Harris, M Rice, Synchronization in software radios. Carrier and timing recovery using FPGAs, in 2000 IEEE Symposium on Field-Programmable Custom Computing Machines (IEEE, Piscataway, 2012), pp. 195-204

29. FJ Harris, M Rice, Multirate digital filters for symbol timing synchronization in software-defined radios. IEEE J. Sel. Areas Commun. 19(12), 2346-2357 (2001)

30. LE Franks, Carrier and bit synchronization in data communication: a tutoria review. IEEE Trans. Commun. 28(8), 1107-1121 (1980)

31. FJ Harris, Performance and design of Farrow filter used for arbitrary resampling. Proc. 13th Int. Conf. DSP 2, 595-599 (1997)

32. CS Burrus, TW Parks, DFT/FFT and Convolution Algorithms: Theory and Implementation (Wiley, New York, 1984)

33. R Shafik, S Rahman, AR Islam, On the extended relationships among EVM, BER and SNR as performance metrics, in International Conference on Electrical and Computer Engineering, 2006. ICECE '06 (IEEE, Piscataway, 2006)

doi:10.1186/1687-6180-2014-62

Cite this article as: Chen et al:: Non-maximally decimated filter bank-based single-carrier receiver: a pathway to next-generation wideband communication. EURASIP Journal on Advances in Signal Processing 2014 2014:62.

\section{Submit your manuscript to a SpringerOpen ${ }^{\odot}$ journal and benefit from:}

- Convenient online submission

- Rigorous peer review

- Immediate publication on acceptance

- Open access: articles freely available online

- High visibility within the field

- Retaining the copyright to your article

Submit your next manuscript at $>$ springeropen.com 